\title{
IgG Fc Glycosylation Patterns of Preterm Infants Differ With Gestational Age
}

\begin{abstract}
Nele Twisselmann ${ }^{1 * \dagger}$, Yannic C. Bartsch ${ }^{2 \dagger}$, Julia Pagel ${ }^{1,3}$, Christian Wieg ${ }^{4}$, Annika Hartz ${ }^{1}$, Marc Ehlers ${ }^{2,5}$ and Christoph Härtel ${ }^{1}$

1 Department of Pediatrics, University of Lübeck and University Medical Center Schleswig-Holstein, Lübeck, Germany, ${ }^{2}$ Laboratories of Immunology and Antibody Glycan Analysis, Institute for Nutrition Medicine, University of Lübeck and University Medical Center Schleswig-Holstein, Lübeck, Germany, ${ }^{3}$ Department of Infectious Diseases and Microbiology, University of Lübeck and University Medical Center Schleswig-Holstein, Lübeck, Germany, ${ }^{4}$ Department of Neonatology, Hospital Aschaffenburg-Alzenau, Aschaffenburg, Germany, ${ }^{5}$ Airway Research Center North (ARCN), German Center for Lung Research (DZL), University of Lübeck, Lübeck, Germany
\end{abstract}

OPEN ACCESS

Edited by:

Nandor Gabor Than,

Hungarian Academy of Sciences

(MTA), Hungary

Reviewed by:

Eszter Tóth

Hungarian Academy of Sciences

(MTA), Hungary

László Drahos,

Hungarian Academy of Sciences

(MTA), Hungary

Josef Cortez,

University of Florida College of

Medicine-Jacksonville, United States

${ }^{*}$ Correspondence:

Nele Twisselmann

nele.twisselmann@uksh.de

†These authors have contributed equally to this work

Specialty section

This article was submitted to Immunological Tolerance and

Regulation,

a section of the journa

Frontiers in Immunology

Received: 29 June 2018 Accepted: 21 December 2018

Published: 18 January 2019

Citation:

Twisselmann N, Bartsch YC, Pagel J,

Wieg $C$, Hartz A, Ehlers $M$ and

Härtel C (2019) IgG FC Glycosylation

Patterns of Preterm Infants Differ With

Gestational Age.

Front. Immunol. 9:3166

doi: 10.3389/fimmu.2018.03166
Preterm infants acquire reduced amounts of Immunoglobulin G (IgG) via trans-placental transfer as compared to term infants which might explain their high susceptibility for infections. The reduced amount of IgG antibodies also results in a lower amount of anti-inflammatory FC N-galactosylated and -sialylated IgG antibodies. This reduction or, even more, a qualitative shift in the type of IgG Fc glycosylation might contribute to the increased risk for sustained inflammatory diseases in preterm infants. It was the aim of our explorative study to investigate the lgG Fc glycosylation patterns in preterm infants of different gestational ages compared to term infants and mothers of preterm infants. In plasma samples of preterm infants $(n=38)$, we investigated IgG concentrations by use of ELISA. Furthermore, we analyzed IgG Fc glycosylation patterns in plasma of preterm infants ( $n=86,23-34$ weeks of gestation), term infants $(n=15)$ and mothers from preterm infants $(n=41)$ using high performance liquid chromatography. Extremely low gestational age infants (born $<28$ weeks of gestation during second trimester) had reduced IgG concentrations and decreased proportions of galactosylated (84.5 vs. $88.4 \%$ ), sialylated (14.5 vs. $17.9 \%$ ) and bisecting $\mathrm{N}$-acetylglucosamine-containing (8.4 vs. $10.8 \%$ ) lgG Fc N-linked glycans as compared to preterm infants born $\geq 28$ weeks of gestation (during third trimester) and term infants. Increased non-galactosylated (agalactosylated, 16.9 vs. 10.6\%) IgG Fc N-linked glycans were associated with the development of chronic inflammatory bronchopulmonary dysplasia (BPD). However, mothers of preterm infants born during second or third trimester of pregnancy did not show significant differences in IgG Fc glycosylation patterns. Thus, the IgG Fc glycosylation patterns of preterm infants depend on their gestational age. Although lack of bisecting $\mathrm{N}$-acetylglucosamine has been associated with less inflammatory effector functions, the decreased IgG Fc galactosylation and sialylation with lower gestational age suggest a rather pro-inflammatory pattern. The difference in IgG Fc glycosylation patterns between preterm infants and mothers of preterm infants suggests a selective enrichment of lgG glyco forms in preterm infants, which might contribute to or result of the development of sustained inflammatory diseases like BPD.

Keywords: newborn, trans-placental transfer, IgG antibodies, IgG Fc glycosylation, mothers, galactosylation, sialylation, preterm infants 


\section{INTRODUCTION}

The adaptation of women during pregnancy leads to a fetomaternal immune tolerance, which is disrupted in the settings of preterm birth. Reasons for preterm birth are often associated with rather pro-inflammatory conditions at the feto-maternal interface (e.g., infection, age, stress) (1). With a compromised and immaturely developed immune system, preterm infants are exposed to the extrauterine environment leading to a high susceptibility for infections and sustained lung inflammation, such as bronchopulmonary dysplasia (BPD) of particular very early preterm infants (2-4).

The endogenous Immunoglobulin $\mathrm{G}(\mathrm{IgG})$ production in infants starts within 1-3 months after term birth (5). Therefore, specific immune protection of infants against pathogens is provided by the active transport of $\operatorname{IgG}$ through the placenta probably exclusively via the neonatal $\mathrm{Fc}$ receptor $(\mathrm{FcRn})(6-10)$. However, IgG concentrations of the fetus are largely diminished in the second trimester of pregnancy. Specifically, during week $17-22$ of gestation only $5-10 \%$ of maternal IgG concentrations are transported via the placenta, which rises to $50 \%$ during week 28-32 of gestation (11). Hence, reduced IgG concentrations in preterm infants might contribute to their predisposition for infection (9).

In addition, a resulting reduced total amount of antiinflammatory galactosylated and sialylated IgG antibodies (12) or, even more, qualitative changes in the type of IgG Fc glycosylation might have an impact on the specific risk profile of preterm infants including inflammation-mediated diseases. IgG Fc glycosylation patterns are characterized as follows: IgG molecules bare an $\mathrm{N}$-glycosylation site on the conserved asparagine at position 297 (N297) of each heavy chain $\mathrm{CH} 2$ domain. The glycan is composed of a biantennary core heptasaccharide comprised of four $\mathrm{N}$-acetylglucosamines (GlcNAc) and three mannoses (Figure 1A). The core structure can be further modified by addition of fucose, bisecting GlcNAc (bisection), galactose (G), or sialic acid (S) (13, 14).

IgG Fc glycosylation patterns are clinically relevant, as a shift toward more non-galactosylated (agalactosylated; G0) glycans has been linked to inflammation-mediated immune diseases (15-19). Animal models have verified that the absence or presence of galactose and sialic acid themselves can influence the inflammatory properties of IgG, i.e., pro-inflammatory [high proportion of agalactosylated glycan structures; $(19,20)]$ or antiinflammatory [high proportion of galactosylated and sialylated glycan structures; $(19,21-27)]$. IgG Fc glycosylation patterns without bisecting GlcNAc have been associated with reduced affinity to classical Fc $\gamma$ receptors and instead less inflammatory conditions (28). The IgG Fc glycosylation pattern thereby influences not only the effector function of antigen-specific IgG in form of immune complexes, but also the immune modulatory effect of total IgG $(12,19,21-26)$.

In humans, IgG Fc glycosylation patterns are variable and influenced by several factors, such as genetics, gender, age, and disease state (29-31). During pregnancy, the degree of galactosylation and sialylation of $\mathrm{IgG}$ antibodies increases whereas the degree of bisecting GlcNAc slightly decreases

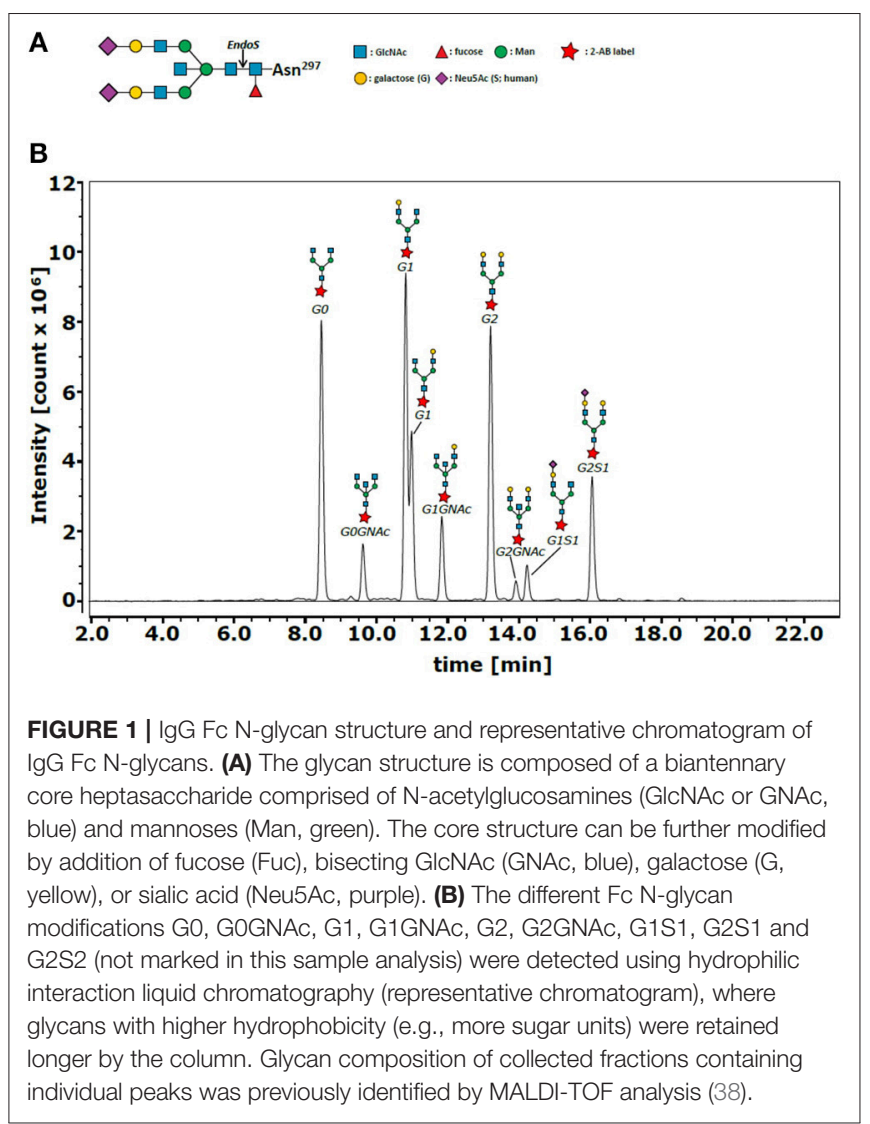

(32-36), which may contribute to the tolerance at the fetomaternal interface. Recent data from cord blood samples have confirmed that the proportion of galactosylated and sialylated IgG is higher in infants at term birth compared to older children as expected from the IgG Fc glycosylation pattern of pregnant women (37).

However, the IgG Fc glycosylation patterns of preterm infants, which are highly susceptible to infection and sustained inflammation, have not been studied yet. It was the primary objective of our explorative study to investigate the Fc glycosylation patterns in the context of preterm birth. We hypothesized that IgG Fc glycosylation patterns of preterm infants are comparable to their mother's type of IgG Fc glycosylation (feto-maternal tolerance) or are polarized toward more inflammatory properties, which might contribute to or result of the development of sepsis and sustained lung inflammation.

\section{MATERIALS AND METHODS}

\section{Study Cohort}

We performed an explorative study in two tertiary care centers for neonates as part of our Immunoregulation of the Newborn (IRoN) study. Samples and clinical data were obtained from infants born between January 1st, 2012 and May 1st, 2015 (center 1; analyzed plasma IgG concentration of infants at mean \pm SD $23 \pm 2$ days of life) and October 1st, 2014 and August 
1st, 2017 (center 2; analyzed IgG Fc glycosylation at mean \pm SD $32 \pm 5$ days of life). The inclusion criteria were preterm infants with gestational age $\geq 23.0$ and $\leq 35.0$ weeks without lethal abnormalities, and written informed consent provided by parents or a legal representative. At center 2 (IgG Fc glycosylation), term infants served as controls (blood withdrawal with routine newborn screening at $48-72 \mathrm{~h}$ of life). Form mothers of preterm infants born in center 2 , we were able to obtain maternal blood from a routine sampling immediately after birth.

\section{Ethics}

Written informed consent was obtained from parents on behalf of the infants enrolled into our studies. The study parts were approved by the local committee on research in human subjects at the University of Lübeck (center 2) and by the local committee on research in human subjects at Ärztekammer Bayern (center 1), respectively. All blood samples were obtained within a medically required blood withdrawal procedure. The additional blood volume obtained for research purposes $(<1 \%$ of whole body blood volume per blood sampling) was in line with current guidelines of the European Medical Agency on the investigation of medicinal products in term and preterm infants; Committee for Medicinal Products for Human Use and Pediatric Committee (39).

\section{Definitions}

Gestational age was calculated from the best obstetric estimate based on early prenatal ultrasound and obstetric examination.

Early-onset sepsis (EOS) was defined as sepsis, clinical or culture-proven, occurring within the first $72 \mathrm{~h}$ of life.

Late-onset sepsis (LOS) was defined as sepsis, clinical or culture-proven, occurring after the first $72 \mathrm{~h}$ of life.
Clinical sepsis was defined as condition when neonatologists decided to treat the infant with antibiotics and continued for at least 5 days due to the following reasons: $\geq 2$ clinical signs of systemic inflammatory response: temperature $>38^{\circ} \mathrm{C}$ or $<36.5^{\circ} \mathrm{C}$, tachycardia $>200 / \mathrm{min}$, new onset or increased frequency of bradycardias or apneas, hyperglycemia $>140 \mathrm{mg} / \mathrm{dl}$, base excess $<-10 \mathrm{mval} / \mathrm{l}$, changed skin color, increased oxygen need; and at least one laboratory sign: C-reactive protein $>10$ $\mathrm{mg} / \mathrm{L}$, platelet count $<100 / \mathrm{nl}$, immature/total neutrophil ratio $>0.2$, white blood cell count $<5 / \mathrm{nl}$ (NeoKISS).

Blood culture-confirmed sepsis was defined as clinical sepsis with proof of causative agent in the blood culture.

Necrotizing enterocolitis (NEC) and focal intestinal perforation (FIP) were defined as surgery due to spontaneous intestinal perforation or necrotizing enterocolitis (Bell stage $\geq 2$ ).

Intraventricular hemorrhage (IVH) was defined according to Papile ultrasound criteria.

Bronchopulmonary dysplasia (BPD) was defined as need for oxygen supplement and/or respiratory support at corrected age of 36 weeks.

Cause of preterm delivery was determined at the discretion of the attending obstetrician, specifically: (1) preterm labor (labor refractory to tocolytic agents) or amniotic infection syndrome [AIS; labor \pm rupture of membranes, maternal fever $\left(\geq 39.0^{\circ} \mathrm{C}\right)$, and/or one of the following signs: increased maternal inflammatory markers without any other cause (CRP $>10 \mathrm{mg} / \mathrm{l}$ or elevation of white blood cell count $>15.000 / \mu \mathrm{l})$, fetal tachycardia, painful uterus and foul-smelling cervical discharge]; (2) pre-eclampsia (pregnancy-induced maternal hypertension, oedema, proteinuria), pathological Doppler (e.g., Arteria umbilicalis Doppler, Ductus venosus flow, Arteria cerebri media Doppler), intrauterine growth restriction as diagnosed by the attending specialist for antenatal ultrasound,

TABLE 1 | Summary of patient demographics from center 1 (IgG quantification).

\begin{tabular}{|c|c|c|c|}
\hline & $<28$ weeks & $\geq 28$ weeks & Total \\
\hline$n$ & 19 & 19 & 38 \\
\hline Gestational age [weeks] & $26.4 \pm 1.0$ & $30.9 \pm 1.9$ & $28.7 \pm 2.7$ \\
\hline Male Gender & $11(58)$ & $8(42)$ & $19(50)$ \\
\hline Multiple & $5(26)$ & $6(32)$ & $11(29)$ \\
\hline NEC & $0(0)$ & $0(0)$ & $0(0)$ \\
\hline FIP & 1 (5) & $0(0)$ & $1(3)$ \\
\hline $\mathrm{IVH}$ & $4(21)$ & $2(11)$ & $6(16)$ \\
\hline BPD & $11(58)$ & $3(16)$ & $14(37)$ \\
\hline \multicolumn{4}{|l|}{ Cause of delivery } \\
\hline
\end{tabular}

EOS, early-onset sepsis; LOS, late-onset sepsis; NEC, necrotizing enterocolitis; FIP, focal intestinal perforation; IVH, intraventricular hemorrhage; BPD, bronchopulmonary dysplasia; AIS, amnion infection syndrome. Data are described as mean \pm standard deviation or $n(\%)$. 
or placental abruption; and (3) others, including cholestasis, etc.

\section{Sample Collection}

Peripheral blood (EDTA) samples were stored at room temperature and processed within $24 \mathrm{~h}$ after withdrawal. Samples were spun down for $6 \mathrm{~min}$ at 1,500 $\mathrm{rpm}$. The clear plasma fraction was transferred and immediately stored at $-80^{\circ} \mathrm{C}$ until further use.

\section{IgG ELISA}

A 96 well plate was coated with anti-human IgG-Fc primary antibody (Bethyl, Laboratories, Montgomer, TX). After blocking residual binding sites, plasma dilutions were added. Intravenous immunoglobulin (IVIG, Biotech, Dreieich, Germany) was used as standard. Plasma IgG concentration was detected with horse radish peroxidase-conjugated anti-human-IgG-Fc antibody (Bethyl, Laboratories, Montgomer, TX) and assay developed with TMB substrate (BD Biosciences).

\section{IgG Purification and IgG-Fc Glycan Analysis}

Total IgG was purified from human plasma samples using Protein G coupled agarose beads (Genetex, San Antonio, TX) in a 96 well filter plate (Merck, Darmstadt, Germany). In brief, 50 $\mu \mathrm{l}$ settled beads were constituted by washing 3 times with $200 \mu \mathrm{l}$

TABLE 2 | Summary of patient demographics from center 2 (IgG Fc glycosylation; infants and mothers of preterm infants).

\begin{tabular}{|c|c|c|c|c|}
\hline & $<28$ weeks & $\geq 28$ weeks & Term infants & Total \\
\hline \multicolumn{5}{|l|}{ INFANTS } \\
\hline$n$ & 43 & 43 & 15 & 101 \\
\hline Gestational age [weeks] & $26.1 \pm 1.2$ & $29.8 \pm 1.6$ & $39.6 \pm 1.2$ & $29.7 \pm 4.7$ \\
\hline Birth weight [g] & $809 \pm 232$ & $1260 \pm 229$ & $3220 \pm 656$ & $1359 \pm 871$ \\
\hline Male Gender & $26(60)$ & $18(42)$ & $8(53)$ & $52(51)$ \\
\hline LOS & $22(51)$ & $6(14)$ & $0(0)$ & $28(28)$ \\
\hline NEC & $2(9)$ & $0(0)$ & $0(0)$ & $2(2)$ \\
\hline FIP & $4(9)$ & $0(0)$ & $0(0)$ & $4(4)$ \\
\hline $\mathrm{IVH}$ & $12(28)$ & 2 (5) & $0(0)$ & $14(14)$ \\
\hline BPD & $14(33)$ & $7(16)$ & $0(0)$ & $21(21)$ \\
\hline Others & $6(14)$ & $3(7)$ & $13(87)$ & $22(22)$ \\
\hline \multicolumn{5}{|l|}{ MOTHERS } \\
\hline$n$ & 16 & 25 & & 41 \\
\hline Age of mother at birth [years] & $33 \pm 6$ & $32 \pm 4$ & & $32 \pm 5$ \\
\hline Gestational age [weeks] & $25.8 \pm 1.6$ & $31.2 \pm 2.1$ & & $29.1 \pm 3.3$ \\
\hline Birth weight [g] & $756 \pm 279$ & $1484 \pm 511$ & & $1200 \pm 562$ \\
\hline Male Gender & $11(69)$ & $11(44)$ & & $22(54)$ \\
\hline Multiple & $2(13)$ & $4(16)$ & & $6(15)$ \\
\hline EOS & $7(44)$ & $2(8)$ & & $9(22)$ \\
\hline LOS & $9(56)$ & $1(4)$ & & $10(24)$ \\
\hline NEC & $2(13)$ & $0(0)$ & & $2(5)$ \\
\hline \multicolumn{5}{|l|}{ MOTHER-INFANT-PAIRS } \\
\hline$n$ & 12 & 8 & 0 & 20 \\
\hline
\end{tabular}

EOS, early-onset sepsis; LOS, late-onset sepsis; AIS, amnion infection syndrome; NEC, necrotizing enterocolitis; FIP, focal intestinal perforation; IVH, intraventricular hemorrhage; BPD, bronchopulmonary dysplasia. Data are described as mean \pm standard deviation or $n(\%)$. 
of PBS and applying negative pressure on a vacuum manifold. Protein G was than incubated with $200 \mu \mathrm{l}$ of a 1:4 plasma dilution (50 $\mathrm{\mu l}$ plasma volume) for $2 \mathrm{~h}$ at room temperature

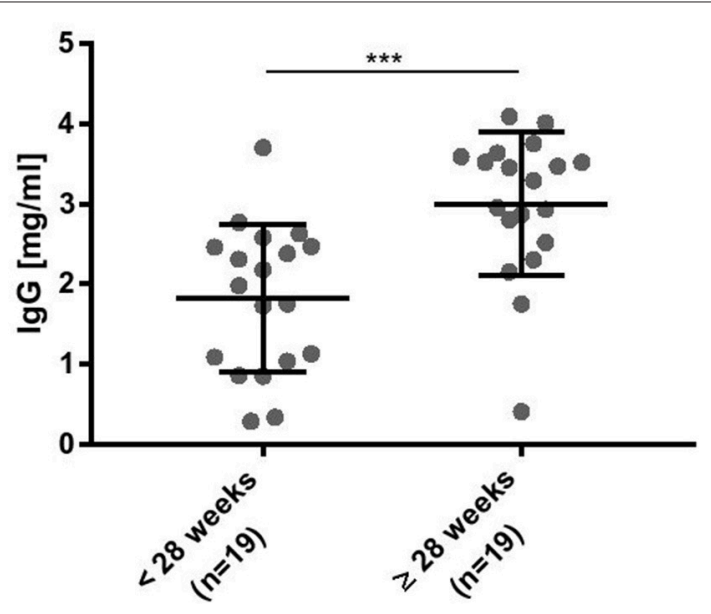

FIGURE 2 | Plasma IgG concentrations were significantly decreased in preterm infants born at lower gestational ages. ELISA data for detection of human IgG-Fc parts. Intravenous immunoglobulin (IVIG) was used as a standard to estimate the plasma lgG concentration (mean $\pm S D$,

Mann-Whitney test). ${ }^{\star \star \star} p<0.001$. with agitation. Unspecific plasma proteins were washed away 5 times with $200 \mu$ l of PBS. IgG was eluted three times with $100 \mu \mathrm{l}$ of $100 \mathrm{mM}$ formic acid ( $\mathrm{pH} 2.5)$. Elution was neutralized by adding $10 \mu \mathrm{l}$ of $1 \mathrm{M}$ ammonium bicarbonate into each elution fraction. IVIG (Biotech, Dreieich, Germany) was used as standard.

From the purified IgG, Fc N-glycan composition was analyzed as previously described (38). Briefly, Fc N-linked glycans were enzymatically released with recombinant endoglycosidase S (EndoS) from Streptococcus pyogenes. EndoS hydrolyses specifically the IgG Fc N-glycan of IgG after the first GlcNAc (Figure 1A). The N-glycans were purified using self-made graphitized carbon columns (Fisher Scientific, Hampton, NH) and labeled with anthranilamide (Sigma-Aldrich). The labeled glycans were analyzed by hydrophilic interaction liquid chromatography-high performance liquid chromatography (HPLC) on a Dionex Ultimate 3000 (Thermo Fischer Scientific, Waltham, Mass) by using an Xbridge XP BEH Glycan column $(1.7 \mu \mathrm{m}$, $100 \times 2.1 \mathrm{~mm}$ i.d.; Waters, Milford, Mass). As previously described (38), glycan composition was identified by MALDI-TOF analysis of collected fractions containing individual peaks. N-glycans with higher hydrophobicity (e.g., more sugar units) were retained longer in the column (Figure 1B).
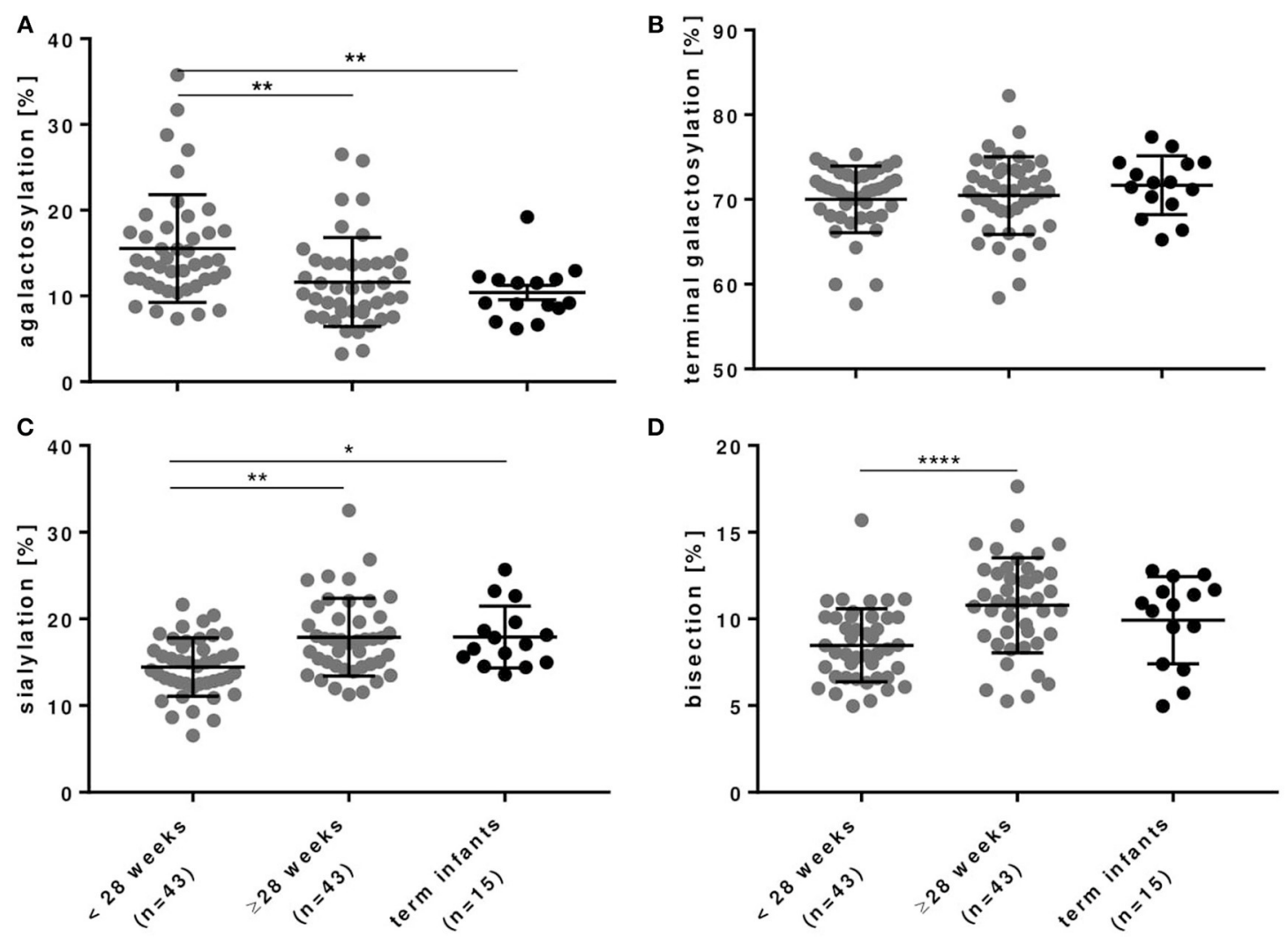

FIGURE 3 | IgG Fc glycosylation in preterm infants is dependent on gestational age. (A) IgG with agalactosylated Fc glycans were significantly increased, whereas (B) IgG with terminally galactosylated Fc glycans were unchanged and IgG with (C) sialylated and (D) bisecting Fc glycans were significantly decreased in preterm infants born $<28$ weeks of gestational age. Term infants served as controls (mean $\pm \mathrm{SD}$, Kruskal-Wallis test). ${ }^{\star} p<0.05,{ }^{\star \star} p<0.01,{ }^{\star \star \star} p<0.0001$. 
The area under the curve (AUC) of the following nine glycan peaks were identified: G0, G0GlcNAc, G1, G1GlcNAc, G2, G2GlcNAc, G1S1, G2S1, and G2S2. Sialylated glycans with bisecting GlcNAc were not detected. The relative proportion of the individual peaks of a sample was calculated by dividing AUC of the individual curve by the AUC of the sum of all nine identified peaks and this multiplied by 100 (for raw data see Supplementary Tables 1, 2). For presentation, the four following groups were defined and percentages of the groups calculated as the sum of the percentages of the individual glycan proportions: (1) agalactosylation: G0 \pm bisecting GlcNAc; (2) terminal galactosylation: G1 \pm bisecting GlcNAc, G2 \pm bisecting
GlcNAc; (3) sialylation: G1S1, G2S1, G2S2; (4) bisection: G0 + bisecting GlcNAc, G1 + bisecting GlcNAc, G2 + bisecting GlcNAc.

\section{Statistical Analysis}

All data were analyzed using GraphPad Prism ${ }^{\circledR}$ version 7. After testing for normal distribution, Kruskal-Wallis test followed by Dunn's multiple comparisons test or the Mann-Whitney test was used for not normally distributed data. Correlations were evaluated using Spearman correlation. Two-way ANOVA followed by Bonferroni's multiple comparison test was done for correlation with clinical parameter. The threshold for

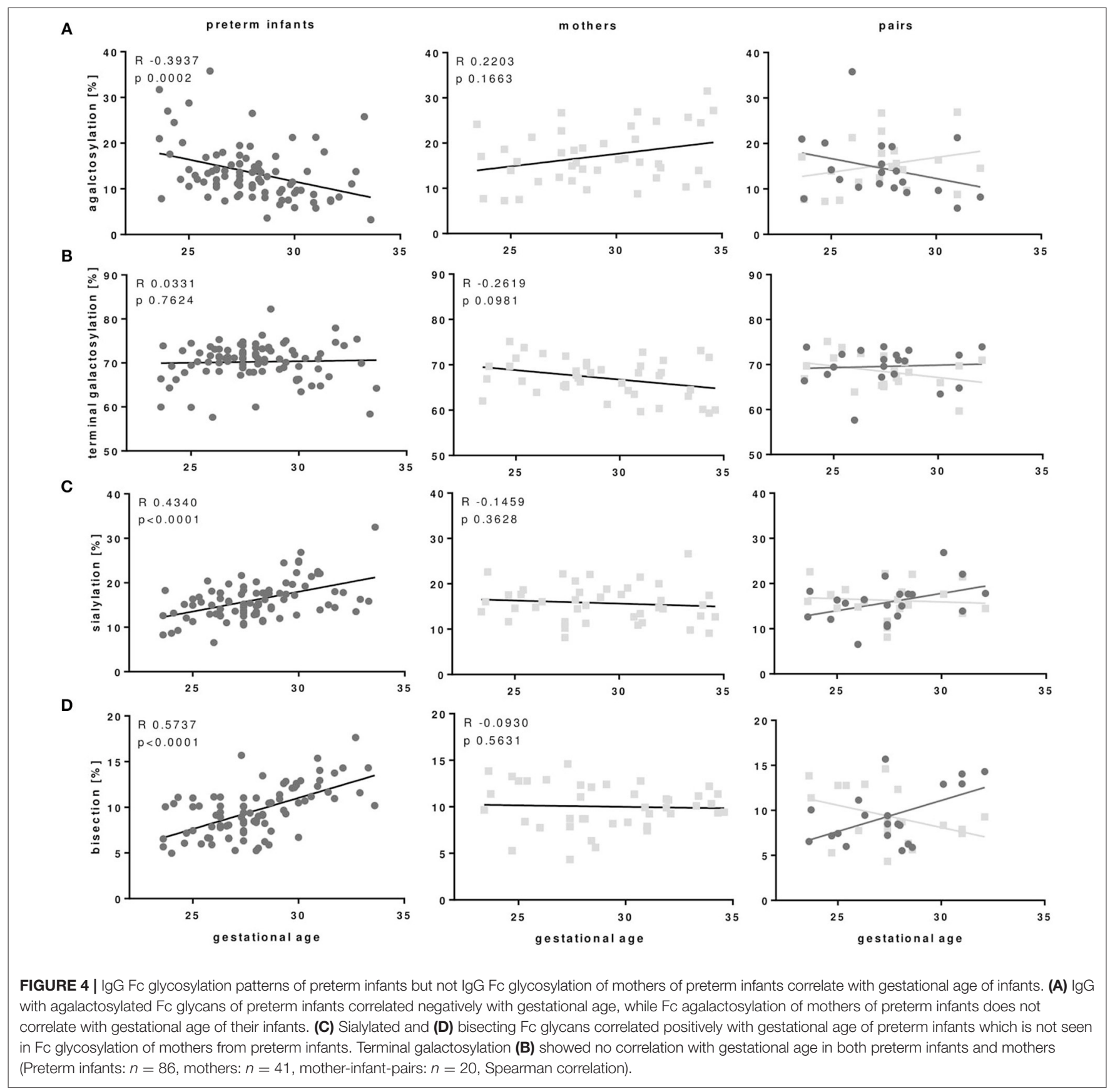


significance was a $p$-value $<0.05$ depicted as ${ }^{*},<0.01$ as $* *,<0.001$ as ${ }^{* * *}$, and $<0.0001$ as $* * * *$.

\section{RESULTS}

\section{Clinical Characteristics}

We recruited a cohort of preterm infants in two centers and collected plasma from peripheral blood samples and key clinical outcome parameters. In samples of center 1 we analyzed plasma IgG concentrations and in samples of center 2 we

TABLE 3 | IgG Fc glycosylation patterns in infants of different gestational age groups depicted in Figure $\mathbf{3}$ (mean $\pm \mathrm{SD}$ ).

\begin{tabular}{lccc}
\hline & $<\mathbf{2 8}$ weeks & $\mathbf{2} \mathbf{2 8}$ weeks & Term infants \\
\hline Agalactosylation (\%) & $15.5 \pm 6.3$ & $11.6 \pm 5.2$ & $10.4 \pm 3.3$ \\
Galactosylation (\%) & $84.5 \pm 6.3$ & $88.4 \pm 5.2$ & $89.6 \pm 3.3$ \\
Terminal galactosylation (\%) & $70.0 \pm 3.9$ & $70.5 \pm 4.6$ & $71.7 \pm 3.5$ \\
Sialylation (\%) & $14.5 \pm 3.4$ & $17.9 \pm 4.5$ & $17.9 \pm 3.6$ \\
Bisection (\%) & $8.4 \pm 2.1$ & $10.8 \pm 2.7$ & $9.9 \pm 2.5$
\end{tabular}

investigated IgG Fc glycosylation. To analyze whether plasma IgG concentrations and Fc glycosylation patterns differ with gestational age, we divided the cohort in 2 gestational age groups (center $1 /$ center 2 : $\geq 23(+0$ days) and $\leq 27(+6)$ weeks, $n=19 / n$ $=43$; $\geq 28(+0)$ and $\leq 34(+6)$ weeks, $n=19 / n=43$; Tables 1, 2).

The cohort of center 1 (plasma IgG concentration) was also selected for a matched pair analysis of BPD diagnosis vs. no BPD. For this matched pair analysis early onset sepsis cases were excluded, and late onset sepsis cases were equal in both groups. In center 2 , we also analyzed IgG Fc glycosylation patterns of term infants $(n=15)$ and mothers of preterm infants $(n=$ 41) (Table 2). For a subgroup of preterm infants and mothers from center 2 , we were able to analyze mother-infant-pairs $(n=20)$.

\section{Plasma IgG Concentrations Were Decreased in Preterm Infants Born at Lower Gestational Ages}

As outlined in Figure 2, IgG was detectable in infants born $<28$ weeks of gestational age and increased significantly with

TABLE 4 | IgG Fc glycosylation patterns of preterm infants associated with (i) gender and clinical parameters defining (ii) cause of birth, (iii) bronchopulmonary dysplasia (BPD) or (iv) sepsis (early and late onset sepsis cases included) (mean \pm SD, two-way ANOVA, multiple comparison within group to adjust for gestational age).

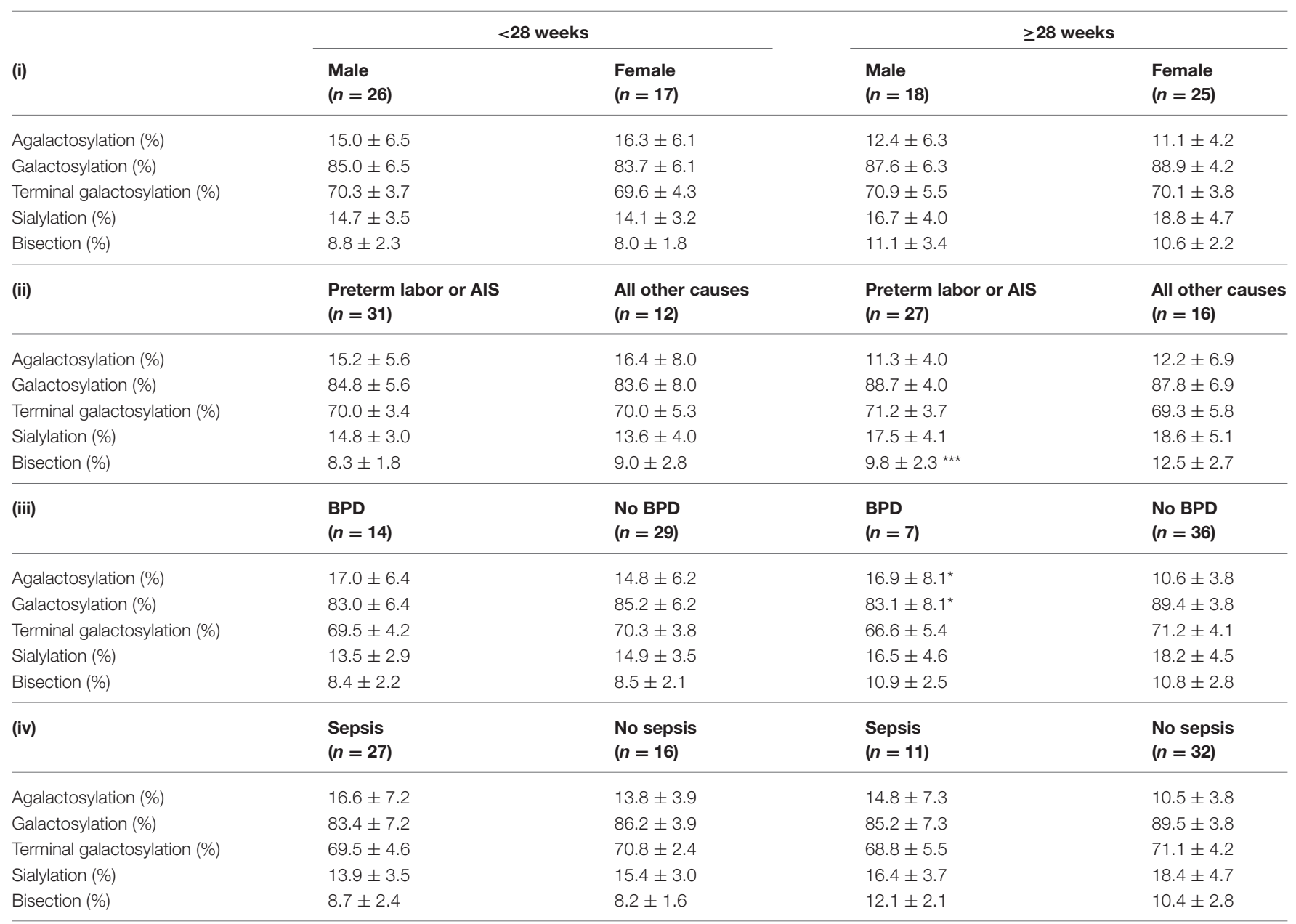

IgG FC agalactosylation is additionally depicted in Figure 5. ${ }^{*} p<0.05,{ }^{* * *} p<0.001$. 
gestational age. Plasma IgG concentrations were not different in a matched pair analysis of preterm infants developing BPD vs. no $\mathrm{BPD}$ diagnosis (data not shown).

\section{IgG Fc Glycosylation Patterns Depend on Gestational Age of Preterm Infants}

As outlined in Figures 3, 4 and Table 3, preterm infants had a higher proportion of agalactosylated (accordingly lower proportion of galactosylated) IgG Fc N-glycans with decreasing gestational age $(R=-0.3937, p=0.0002)$. No difference in agalactosylated IgG Fc glycans was observed between infants born $\geq 28$ weeks of gestation and term controls. Further, no correlation between terminal galactosylation of IgG and gestational age in preterm infants was found $(R=0.0331, p=$ 0.76). However, also IgG Fc sialylation was significantly lower in preterm infants born $<28$ weeks as compared to infants born $\geq 28$ weeks of gestation and term infants (correlation with gestational age; $R=0.4340, p<0.0001)$. The proportion of IgG glycans with bisecting GlcNAc also correlated with gestational age $(R=$ 0.5737, $p<0.0001)$.

\section{IgG Fc Glycosylation Shows Differences Between Preterm Infants and Mothers of Preterm Infants}

In the comparison of maternal with neonatal IgG Fc glycosylation patterns we did not find the same correlation between IgG Fc glycosylation patterns in mothers of preterm infants with the gestational age of their infants which we observed between the IgG Fc glycosylation patterns of preterm infants with their gestational age (Figure 4).

\section{Clinical Correlation of IgG Fc Glycosylation in Preterm Infants}

For the cause of preterm delivery bisecting IgG Fc glycosylation was significantly decreased in preterm infants born $\geq 28$ weeks of gestation with preterm labor or amnion infection syndrome (AIS) as compared to other preterm delivery reasons (Table 4).

For the development of BPD, preterm infants born $<$ or $\geq 28$ weeks of gestation had respectively, a tending or significant increased proportion of agalactosylated IgG as compared to preterm infants without BPD (Figure 5 and Table 4). A similar

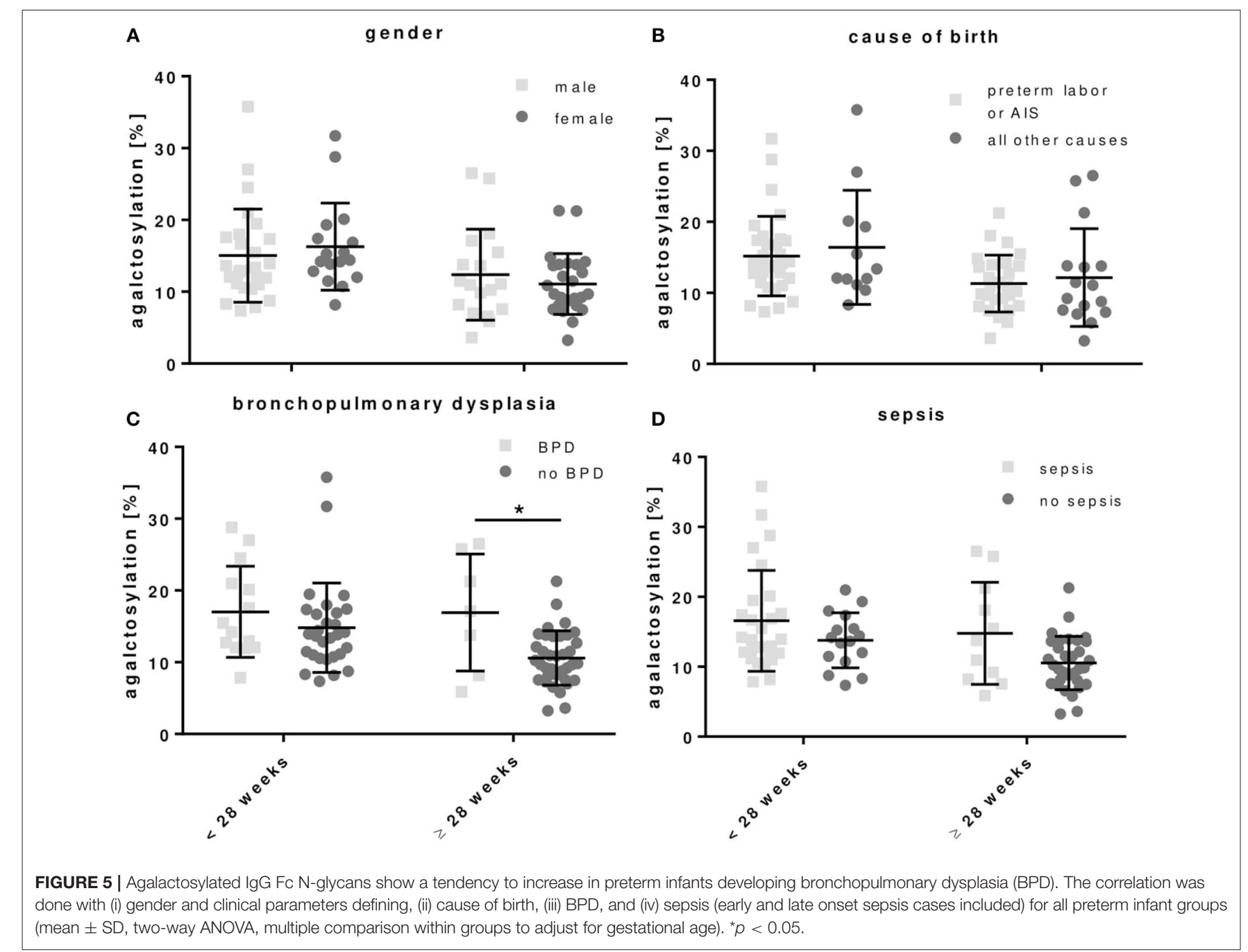


tendency, which was however not significant, was observed in preterm infants with sepsis (Figure 5 and Table 4). No differences in IgG Fc glycosylation were found for the gender (Figure 5 and Table 4).

\section{DISCUSSION}

To our knowledge, this is the first explorative study investigating IgG Fc glycosylation patterns in peripheral blood of preterm infants. We noted that IgG Fc glycosylation patterns of preterm infants differ with gestational age whereas the patterns of mothers, as described in the literature $(32,33)$, did not differ with gestational age of their offspring. Preterm infants born $<28$ weeks of gestation displayed an IgG Fc glycosylation pattern characterized by a higher proportion of agalactosylated IgG (reduced proportion of galactosylated IgG) and a reduced proportion of sialylated and bisecting IgG. The pattern of Fc glycosylation from mothers, however, did not differ between the second and third trimester of pregnancy suggesting an enrichment of IgG glyco forms in extremely low gestational age infants. This novel finding needs further prospective in-depthanalysis, how these IgG Fc glyco forms are enriched in the smallest, most vulnerable infants, whether they contribute to or are a result of dysregulated immune responses and sustained inflammation like BPD, and how they function.

We were able to confirm that IgG antibodies are transported through the placenta during second trimester of pregnancy, but in a much less amount in infants born $<28$ weeks of gestation as compared to infants born $\geq 28$ weeks of gestation. In our setting, IgG concentration of preterm infants $\geq 28$ weeks of gestation were still below the levels of term born infants (clinical reference values for infants between 1 and 3 months of age are 2.5-7.5 $\mathrm{mg} / \mathrm{ml} \mathrm{IgG,} \mathrm{Immunology} \mathrm{laboratory} \mathrm{of} \mathrm{the} \mathrm{medical} \mathrm{clinic} \mathrm{in}$ Freiburg, Germany, 2007).

Our current understanding of IgG Fc glycosylation patterns and its functional role for the immune system is that different Fc N-linked glycan structures can influence the inflammatory properties of IgG antibodies. Galactosylation and sialylation of total serum IgG but also of antigen-specific IgG in form of immune complexes were shown to mediate more antiinflammatory effector functions $(12,19,21-27)$ whereas a shift toward more agalactosylated and bisecting glycan structures has been associated with pro-inflammatory effects $(19,20,28)$. IgG glycosylation of pregnant women in the Fc part was shown to be similar during second and third trimester of pregnancy and directed toward an anti-inflammatory pattern as compared to non-pregnant woman $(32,33)$. This pattern was also previously found in cord blood samples of term infants $(35,37)$ and was suggested to contribute to the condition of feto-maternal tolerance and permissive microbiota establishment after birth. In the context of preterm birth, however, the IgG Fc glycosylation patterns are different between infants born during second and third trimester and it is yet unknown whether immune dysregulation derives from a pro-inflammatory setting, i.e., reduced galactosylation and sialylation, or from an antiinflammatory effect due to reduced bisection leading to lower affinities to activating Fc $\gamma$ receptors. For this reason, functional studies are needed determining which IgG Fc glycosylation pattern is dominant. We showed that reduced bisection was associated with birth pathology, especially preterm labor and amnion infection syndrome (AIS) suggesting a role of $\mathrm{Fc}$ glycosylation patterns for preterm birth. Additionally, we showed a trend of increased IgG Fc agalactosylation in inflammatorymediated diseases of preterm infants (i.e., chronic lung disease) supporting the hypothesis of a pro-inflammatory effect.

Since IgG of infants in the first month of life are mainly of maternal origin (5), several studies investigated the role of trans-placental transfer through $\mathrm{FcRn}$ to be selective for certain Fc glycosylation. In vitro, FcRn has a higher affinity to galactosylated IgG (40). A few studies showed a transfer of more galactosylated IgG to infants born at term by comparing IgG glycosylation in healthy pregnant women and umbilical cord blood of term infants $(35,41,42)$. In another study, a similar IgG Fc glycosylation pattern between maternal sample and cord blood was reported (43). In our setting, IgG Fc glycosylation patterns in peripheral blood revealed a difference between preterm infants born during second and third trimester compared to IgG Fc glycosylation of mothers from preterm infants which is not changing over the second and third trimester. The reduced galactosylation, sialylation and bisection in extremely preterm infants might reflect the enrichment of certain IgG glyco forms at lower gestation. Since endogenous IgG production slowly starts at 1-3 months (5), we assumed that detected IgG in preterm infants is still of maternal origin. Several mechanisms for the enrichment of the described IgG glyco forms in preterm infants born $<28$ weeks of gestation might be possible. First, a selective IgG transport via the placenta might play a role in early stages of pregnancy. Second, trimming (44) or extracellular modification (45) of the sugars from IgG Fc glycans in the circulation of early preterm infants might result in a dominance of the detected IgG Fc glycosylation pattern. Third, IgG in breast milk might modify the IgG Fc glycosylation pattern also it only contains about $0.01-0.06 \mathrm{mg} / \mathrm{ml}$ in women from western countries (46).

Taken together, our findings suggest an enrichment of certain IgG glyco forms in extremely low gestational age infants and that a high proportion of "pro-inflammatory" agalactosylated IgG Fc N-glycans in those infants might contribute to or result from inflammation-mediated diseases. However, IgG Fc agalactosylation was not significantly associated with the diagnosis of BPD or sepsis in extremely preterm infants, only with the diagnosis of BPD in preterm infants born $\geq 28$ weeks of gestation. Future studies need to evaluate larger cohorts using multivariable linear regression accounting for possible confounding factors. Furthermore, functional in vitro and in vivo studies are needed to reveal consequences for effector functions of IgG glycosylation pattern in term and preterm infants.

The use of peripheral blood from infants in contrast to umbilical cord blood has the advantage not to be contaminated with maternal blood in the context of clinical sampling. Despite the limited sample volumes, we obtained peripheral blood samples of highly vulnerable infants. Our data are hypothesisgenerating and add another component to the discussion of intravenous substitution of pooled immunoglobulins 
(IVIg) to preterm infants. Large trials have shown no benefit (INIS trial) with regard to sepsis risk or neurodevelopmental outcome (47), but to e.g., reduction of the inflammatory cytokine IL-6 (48). However, the role of IgG Fc glycosylation has never been reflected in the context of IVIg treatment in preterm infants and also not in the context of BPD. The effect might be beneficial in sustained inflammation of preterm infants, if the intravenous immunoglobulins would be immunomodulatory with higher proportions of galactosylation and sialylation, since this would simulate more closely the measured IgG glycosylation patterns in term infants and might mediate anti-inflammatory effects in preterm infants.

In summary, our data suggest that IgG Fc glycosylation patterns differ in preterm infants and their mothers suggesting an enrichment of certain IgG glyco forms in early stages of gestation. Further investigations with a larger cohort are needed to determine the enrichment and functional role of the observed IgG Fc glycosylation pattern in early preterm infants and to verify its correlation with sustained inflammation of preterm infants.

\section{AUTHOR CONTRIBUTIONS}

$\mathrm{NT}, \mathrm{YB}, \mathrm{JP}, \mathrm{AH}, \mathrm{ME}$, and $\mathrm{CH}$ contributed conception and design of the study. $\mathrm{CH}$ and $\mathrm{CW}$ provided clinical samples. $\mathrm{YB}$ performed experiments and HPLC analysis. NT correlated HPLC analysis with clinical data and performed statistical analysis. NT and YB wrote first draft of the manuscript. All authors contributed to manuscript revision, read and approved the submitted version.

\section{REFERENCES}

1. Arck PC, Hecher K. Fetomaternal immune cross-talk and its consequences for maternal and offspring's health. Nat Med Rev. (2013) 19:548-56. doi: $10.1038 / \mathrm{nm} .3160$

2. Stoll BJ, Hansen NI, Bell EF, Walsh MC, Carlo WA, Shankaran S, et al. Trends in care practices, morbidity, and mortality of extremely preterm neonates, 1993-2012. JAMA J Am Med Assoc. (2015) 314:1039-51. doi: 10.1001/jama.2015.10244

3. Niedermaier S, Hilgendorff A. Bronchopulmonary dysplasia-an overview about pathophysiologic concepts. Mol Cell Pediatrics (2015) 2:2. doi: 10.1186/s40348-015-0013-7

4. Kan B, Razzaghian HR, Lavoie PM. An immunological perspective on neonatal sepsis. Trends Mol Med. (2016) 22:290-302. doi: 10.1016/j.molmed.2016.02.001

5. Morell A, Skvaril F, Hitzig WH, Barandun S. IgG subclasses: development of the serum concentrations in 'normal' infants and children. J Pediatr. (1972) 80:960-4.

6. Jauniaux E, Jurkovic D, Gulbis B, Liesnard C, Lees C, Campbell S. Materno-fetal immunoglobulin transfer and passive immunity during the first trimester of human pregnancy. Hum Reprod. (1995) 10:3297-300. doi: 10.1093/oxfordjournals.humrep.a135906

7. Gonçalves G, Cutts FT, Hills M, Rebelo-Andrade H, Trigo FA, Barros H. Transplacental transfer of measles and total IgG. Epidemiol Infect. (1999) 122:273-9. doi: 10.1017/S0950268899002046

8. Firan M, Bawdon R, Radu C, Ober RJ, Eaken D, Antohe F, et al. The MHC class I-related receptor, FcRn, plays an essential role in the maternofetal transfer of $\gamma$-globulin in humans. Int Immunol. (2001) 13:9931002. doi: 10.1093/intimm/13.8.993

\section{FUNDING}

NT was supported by a grant of the Friedrich-EbertStiftung (financed by the German Ministry of Education and Research, BMBF). JP was supported by a grant of the German Centre of Infection Research (DZIF; financed by the German Ministry of Education and Research, BMBF). $\mathrm{AH}$ was supported by grants from the IRTG 1911 (financed by the German Society of Research, DFG). CH was funded by the BMBF (PRIMAL clinical study, No. 01GL1746A), the University of Lübeck and Lübeck-Hilfe für krebskranke Kinder e.V.

\section{ACKNOWLEDGMENTS}

We are indebted to the patients and their families for their participation and the physicians of the local NICU for their generous collaboration in this study. We thank Matthias Collin for the EndoS. The experimental work of ME's laboratories was supported by the Else-Kröner-Fresenius Foundation (2014_A91) and the German Research Foundation (DFG; EH 221/8-1, /91, /10-1, /11-1, Research Training Group (GRK) 1727, Clinical Research Unit (CRU) 303, SFB/TR 654 and Excellence cluster 306 Inflammation at Interfaces).

\section{SUPPLEMENTARY MATERIAL}

The Supplementary Material for this article can be found online at: https://www.frontiersin.org/articles/10.3389/fimmu. 2018.03166/full\#supplementary-material

9. Silveira Lessa AL, Krebs VL, Brasil TB, Pontes GN, Carneiro-Sampaio M, Palmeira P. Preterm and term neonates transplacentally acquire IgG antibodies specific to LPS from Klebsiella pneumoniae, Escherichia coli and Pseudomonas aeruginosa. FEMS Immunol Med Microbiol. (2011) 62:236-43. doi: 10.1111/j.1574-695X.2011.00807.x

10. Fouda GG, Martinez DR, Swamy GK, Permar SR. The impact of IgG transplacental transfer on early life immunity. ImmunoHorizons (2018) 2:1425. doi: 10.4049/immunohorizons.1700057

11. Malek A, Sager R, Kuhn P, Nicolaides KH, Schneider H. Evolution of maternofetal transport of immunoglobulins during human pregnancy. Am J Reprod Immunol. (1996) 36:248-55. doi: 10.1111/j.1600-0897.1996. tb00172.x

12. Maddur MS, Kaveri SV, Bayry J. Circulating normal IgG as stimulator of regulatory $\mathrm{T}$ cells: lessons from intravenous immunoglobulin. Trends Immunol. (2017) 38:789-92. doi: 10.1016/j.it.2017.08.008

13. Arnold JN, Wormald MR, Sim RB, Rudd PM, Dwek RA. The impact of glycosylation on the biological function and structure of human immunoglobulins. Annu Rev Immunol. (2007) 25:21-50. doi: 10.1146/annurev.immunol.25.022106.141702

14. Jefferis R. Glycosylation as a strategy to improve antibody-based therapeutics. Nat Rev Drug Discov. (2009) 8:226-34. doi: 10.1038/nrd2804

15. Ercan A, Cui J, Chatterton DE, Deane KD, Hazen MM, Brintnell W, et al. Aberrant IgG galactosylation precedes disease onset, correlates with disease activity, and is prevalent in autoantibodies in rheumatoid arthritis. Arthritis Rheum. (2010) 62:2239-48. doi: 10.1002/art.27533

16. Wuhrer M, Selman MH, McDonnell LA, Kümpfel T, Derfuss T, Khademi $\mathrm{M}$, et al. Pro-inflammatory pattern of IgG1 Fc glycosylation in multiple sclerosis cerebrospinal fluid. J Neuroinflammation. (2015) 12:235. doi: 10.1186/s12974-015-0450-1 
17. Vučković F, Krištić J, Gudelj I, Teruel M, Keser T, Pezer M, et al. Association of systemic lupus erythematosus with decreased immunosuppressive potential of the IgG glycome. Arthritis Rheumatol. (2015) 67:2978-89. doi: 10.1002/art.39273

18. Trbojević Akmačić I, Ventham NT, Theodoratou E, Vučković F, Kennedy NA, Krištić J, et al. Inflammatory bowel disease associates with proinflammatory potential of the immunoglobulin G glycome. Inflammatory Bowel Dis. (2015) :1237-47. doi: 10.1097/MIB.0000000000000372

19. Bartsch YC, Rahmöller J, Mertes MMM, Eiglmeier S, Lorenz FKM, Stoehr AD, et al. Sialylated autoantigen-reactive IgG antibodies attenuate disease development in autoimmune mouse models of lupus nephritis and rheumatoid arthritis. Front Immunol. (2018) 9:1183. doi: 10.3389/fimmu.2018.01183

20. Van Beneden K, Coppieters K, Laroy W, De Keyser F, Hoffman IE, Van den Bosch F, et al. Reversible changes in serum immunoglobulin galactosylation during the immune response and treatment of inflammatory autoimmune arthritis. Ann Rheum Dis. (2009) 68:1360-5. doi: 10.1136/ard.2008. 089292

21. Kaneko Y, Nimmerjahn F, Ravetch JV. Anti-inflammatory activity of immunoglobulin G resulting from Sc sialylation. Science (2006) 313:670-3. doi: $10.1126 /$ science. 1129594

22. Karsten CM, Pandey MK, Figge J, Kilchenstein R, Taylor PR, Rosas M, et al. Anti-inflammatory activity of IgG1 mediated by Fc galactosylation and association of Fc $\gamma$ RIIB and dectin-1. Nat Med. (2012) 18:1401-6. doi: $10.1038 / \mathrm{nm} .2862$

23. Oefner CM, Winkler A, Hess C, Lorenz AK, Holecska V, Huxdorf M, et al. Tolerance induction with $\mathrm{T}$ cell-dependent protein antigens induces regulatory sialylated IgGs. J Allergy Clin Immunol. (2012) 129:1647-55.e13. doi: 10.1016/j.jaci.2012.02.037

24. Anthony RM, Wermeling F, Ravetch JV. Novel roles for the IgG Fc glycan. Ann $N$ Y Acad Sci. (2012) 1253:170-180. doi: $10.1111 /$ j.1749-6632.2011.06305.x

25. Hess C, Winkler A, Lorenz AK, Holecska V, Blanchard V, Eiglmeier S, et al. T cell-independent B cell activation induces immunosuppressive sialylated IgG antibodies. J Clin Invest. (2013) 123:3788-96. doi: 10.1172/JCI65938

26. Collin M, Ehlers M. The carbohydrate switch between pathogenic and immunosuppressive antigen-specific antibodies. Exp Dermatol. (2013) 22:511-4. doi: 10.1111/exd.12171

27. Lilienthal GM, Rahmöller J, Petry J, Bartsch YC, Leliavski A, Ehlers M. Potential of murine IgG1 and human IgG4 to inhibit the classical complement and Fc $\gamma$ receptor activation pathways. Front Immunol. (2018) 9:958. doi: 10.3389/fimmu.2018.00958

28. Davies J, Jiang L, Pan LZ, LaBarre MJ, Anderson D, Reff M. Expression of GnTIII in a recombinant anti-CD20 CHO production cell line: expression of antibodies with altered glycoforms leads to an increase in ADCC through higher affinity for FC gamma RIII. Biotechnol Bioeng. (2001) 74:288-94. doi: 10.1002/bit.1119

29. Ruhaak LR, Uh HW, Beekman M, Koeleman CA, Hokke CH, Westendorp RG, et al. Decreased levels of bisecting GLcNAc glycoforms of IgG are associated with human longevity. PLoS ONE (2010) 5:e12566 doi: 10.1371/journal.pone.0012566

30. Pučić M, KneŽević A, Vidič J, Adamczyk B, Novokmet M, Polašek O, et al. High throughput isolation and glycosylation analysis of IgG-variability and heritability of the IgG glycome in three isolated human populations. Mol Cell Proteomics (2011) 10:M111.010090. doi: 10.1074/mcp.M111. 010090

31. Dekkers G, Rispens T, Vidarsson G. Novel concepts of altered immunoglobulin $\mathrm{G}$ galactosylation in autoimmune diseases. Front Immunol. (2018) 9:553. doi: 10.3389/fimmu.2018.00553

32. van de Geijn FE, Wuhrer M, Selman MH, Willemsen SP, de Man YA, Deelder AM, et al. Immunoglobulin G galactosylation and sialylation are associated with pregnancy-induced improvement of rheumatoid arthritis and the postpartum flare: results from a large prospective cohort study. Arthritis Res Ther. (2009) 11:R193. doi: 10.1186/ar2892

33. Bondt A, Rombouts Y, Selman MHJ, Hensbergen PJ, Reiding KR, Hazes JMW, et al. Immunoglobulin G (IgG) fab glycosylation analysis using a new mass spectrometric high-throughput profiling method reveals pregnancy-associated changes. Mol Cell Proteomics (2014) 13.11:3029-39. doi: 10.1074/mcp.M114.039537

34. Jansen BC, Bondt A, Reiding KR, Lonardi E, de Jong CJ, Falck D, et al. and Wuhrer M. Pregnancy-associated serum N-glycome changes studied by high-throughput MALDI-TOF-MS. Sci Rep. (2016) 6:23296. doi: 10.1038/srep23296

35. Jansen BC, Bondt A, Reiding KR, Scherjon SA, Vidarsson G, Wuhrer M. MALDI-TOF-MS reveals differential N-linked plasma-and IgG-glycosylation profiles between mothers and their newborns. Sci Rep. (2016) 6:34001. doi: $10.1038 /$ srep34001

36. Bondt A, Hafkenscheid L, Falck D, Kuijper TM, Rombouts Y, Hazes JMW, et al. ACPA IgG galactosylation associates with disease activity in pregnant patients with rheumatoid arthritis. Ann Rheum Dis. (2018) 77:1-7. doi: 10.1136/annrheumdis-2018-212946

37. De Haan N, Reiding KR, Driessen G, Van Der Burg M, Wuhrer M. Changes in healthy human IgG Fc-glycosylation after birth and during early childhood. J Proteome Res. (2016) 15:1853-61. doi: 10.1021/acs.jproteome.6b00038

38. Epp A, Hobusch J, Bartsch YC, Petry J, Lilienthal GM, Koeleman CAM, et al. Sialylation of IgG antibodies inhibits IgG-mediated allergic reactions. J Allergy Clin Immunol. (2018) 141:399-402.e8. doi: 10.1016/j.jaci.2017.06.021

39. Pediatric Committee of the European Union (PDCO) (2006). Available online at: https://www.ema.europa.eu/en/human-regulatory/overview/paediatricmedicines/paediatric-regulation

40. Dashivets T, Thomann M, Rueger P, Knaupp A, Buchner J, Schlothauer T. Multi-angle effector function analysis of human monoclonal IgG glycovariants. PLoS ONE (2015) 10:e0143520. doi: 10.1371/journal.pone.0143520

41. Williams PJ, Arkwright PD, Rudd P, Scragg IG, Edge CJ, Wormald MR, et al. Selective placental transport of maternal IgG to the fetus. Placenta (1995) 16:749-56. doi: 10.1016/0143-4004(95)90018-7

42. Kibe T, Fujimoto S, Ishida C, Togari H, Wada Y, Okada S, et al. Glycosylation and placental transport of immunoglobulin G. J Clin Biochem Nutr. (1996) 21:57-63. doi: 10.3164/jcbn.21.57

43. Einarsdottir HK, Selman MHJ, Kapur R, Scherjon S, Koeleman CAM, Deelder AM, et al. Comparison of the FC glycosylation of fetal and maternal immunoglobulin G. Glycoconj J. (2013) 30:147-57. doi: 10.1007/s10719-012-9381-6

44. Yang WH, Aziz PV, Heithoff DM, Mahan MJ, Smith JW, Marth JD. An intrinsic mechanism of secreted protein aging and turnover. Proc Natl Acad Sci USA. (2015) 112:13657-62. doi: 10.1073/pnas.1515464112

45. Jones MB, Oswald DM, Joshi S, Whiteheart SW, Orlando R, Cobb BA. B-cellindependent sialylation of IgG. Proc Natl Acad Sci USA. (2016) 113:7207-12. doi: $10.1073 /$ pnas. 1523968113

46. Ruiz L, Espinosa-Martos I, García-Carral C, Manzano S, McGuire MK, Meehan CL, et al. What's normal? immune profiling of human milk from healthy women living in different geographical and socioeconomic settings. Front. Immunol. (2017) 8:696. doi: 10.3389/fimmu.2017.00696

47. Ohlsson A, Lacy JB. Intravenous immunoglobulin for suspected or proven infection in neonates. Cochrane Database Syst Rev. (2015) CD001239. doi: 10.1002/14651858.CD001239.pub5

48. Liu P, Li L, Fan P, Zheng J, Zhao D. High-dose of intravenous immunoglobulin modulates immune tolerance in premature infants. BMC Pediatr. (2018) 18:74. doi: 10.1186/s12887-018-1055-5

Conflict of Interest Statement: The authors declare that the research was conducted in the absence of any commercial or financial relationships that could be construed as a potential conflict of interest.

Copyright (c) 2019 Twisselmann, Bartsch, Pagel, Wieg, Hartz, Ehlers and Härtel. This is an open-access article distributed under the terms of the Creative Commons Attribution License (CC BY). The use, distribution or reproduction in other forums is permitted, provided the original author(s) and the copyright owner(s) are credited and that the original publication in this journal is cited, in accordance with accepted academic practice. No use, distribution or reproduction is permitted which does not comply with these terms. 